\title{
Caractérisation non destructive des bétons par propagation d'ondes ultrasonores
}

\author{
Vincent Garnier a , Jean François Chaix, Mathieu Rossat, Cédric Payan \\ et Gilles Corneloup \\ LCND, Université de la Méditerranée, 413 boulevard Gaston Berger, 13625 Aix-en-Provence Cedex 1, France
}

Reçu le 20 avril 2009

\begin{abstract}
Résumé - La caractérisation non destructive des structures en béton par propagation d'ondes ultrasonores permet d'accéder aux vitesses voire atténuations et spectres fréquentiels pour diagnostiquer une pathologie ou un état du matériau. La forte hétérogénéité du béton au regard des longueurs d'ondes conduit à des phénomènes de diffusion et de non linéarité acoustique. Les conséquences sur les paramètres ultrasonores sont analysées tant sur l'aspect propagation que rétrodiffusion.
\end{abstract}

Mots clés : Caractérisation / ondes / béton /diffusion

Abstract - Non destructive characterisation of concretes by ultrasonic wave's propagation. The non destructive characterization of concretes structures with ultrasonic wave's propagation allows determining the velocities and the attenuation or frequencies spectrum. It can be possible to diagnostic a pathology or the material state. The strong concrete heterogeneity regarding induces scattering and non linear acoustic phenomena. The consequences on the ultrasonic parameters are analysed on the forward and backscattered propagation.

Key words: Characterisation / wave's / concrete / scattering

\section{Introduction}

La caractérisation des bétons par propagation d'ondes ultrasonores est une voie usuelle pour évaluer la résistance potentielle d'un ouvrage. L'utilisation de techniques éprouvées telle que la transmission, le pulse écho ou les ondes de surfaces permettent de repérer des zones à faibles caractéristiques mécaniques, voire à détecter la présence de fissures. Toutefois il est important de noter que de nombreux paramètres expérimentaux et environnementaux peuvent influencer les mesures. Il convient d'être très prudent sur les valeurs absolues relevées. Les corrélations vitesse ultrasonore - résistance à la compression qui sont généralement utilisées ne peuvent s'appliquer qu'à des exemples donnés dans des circonstances spécifiques.

La connaissance fine de la propagation des ondes dans le béton et les avantages qu'elle pourra apporter sur le point de vue théorique deviennent essentiels pour envisager des contrôles sur site et faire des choix de procédures ou de paramètres de contrôle. Les phénomènes de diffusion multiple que génère l'hétérogénéité conséquente

a Auteur pour correspondance : du béton sont issus des interactions des ondes ultrasonores avec chaque diffuseur potentiel. Dans un premier temps, nous analysons les possibilités de modéliser les ondes cohérentes diffusées et transmises à travers un matériau théorique le plus proche possible du béton. Nous proposons une démarche théorique ayant pour but d'homogénéiser le matériau et nous analysons les limites d'application de celle-ci en transmission. Les conclusions conduisent à proposer une technique de contrôle simple fondée sur l'exploitation de la partie rétrodiffusée de l'onde par exemple. Celle-ci rassemble l'ensemble du bruit généré par le matériau que nous lions à des caractéristiques du béton. Des essais sur des matériaux réels montrent les développements à mener pour tendre vers l'application industrielle.

\section{Modélisation de la propagation des ondes dans le béton}

Les ondes ultrasonores qui se propagent dans le béton subissent une divergence géométrique due à la morphologie du faisceau, une dispersion sous forme de chaleur 
ainsi que de la diffusion par interaction avec l'ensemble des diffuseurs qu'elles rencontrent. Ce sont les granulats, les grains de sables, les fissures et les microfissures voire les barres de renfort. Les vitesse et atténuation évoluent alors et ceci notamment en fonction de la fréquence. L'endommagement ou l'évolution de certaines pathologies peuvent modifier la distribution, la forme et la densité de ces diffuseurs. Il convient alors de mesurer ou de prédire leurs influences sur les paramètres acoustiques.

Suivant la longueur d'onde $\lambda$ comparée à la taille des diffuseurs $d$, les interactions peuvent être classées en trois domaines qui sont dits de Rayleigh pour $\lambda \gg d$, stochastiques pour $\lambda \approx d$ et géométrique pour $\lambda \ll d$. Le cas du béton avec des longueurs d'ondes utilisées de l'ordre de $100 \mathrm{~mm}$ à moins de $5 \mathrm{~mm}$ pour des fréquences de moins de l'ordre de $20 \mathrm{kHz}$ à plus de $1 \mathrm{MHz}$ se situe dans le domaine stochastique pour lequel les phénomènes de diffusion sont importants. En se limitant à ce dernier phénomène qui modifie la vitesse et l'atténuation des ondes dans l'espace, l'énergie du faisceau peut être transférée en ondes transmises cohérentes, en ondes transmises incohérentes ainsi qu'en ondes rétrodiffusées. Ces dernières seront étudiées en deuxième partie de ce travail. La partie incohérente dite coda est analysée par différentes méthodes. Pour exemple, la constante de diffusion du matériau peut en être déduite, mais les applications sont peu exploitables sur site.

La partie cohérente rentre dans le champ de calculs statistiques qui conduisent à l'homogénéisation du matériau. Le principe général consiste à calculer un matériau de propriétés acoustiques équivalentes à celui du matériau hétérogène constitué de plusieurs matériaux de caractéristiques, de géométries et dimensions connues. Le taux de chaque constituant dans la matrice est maîtrisé.

Waterman et Truell [1] proposent un modèle d'homogénéisation qui permet de calculer la vitesse et l'atténuation du matériau équivalent à partir des données propres des différents constituants. Il prend en compte des diffuseurs moyens. Le champ moyen est alors donné par :

$$
\langle\varphi\rangle=\varphi_{0} \mathrm{e}^{i k^{*} \cdot \vec{r}}
$$

avec $\varphi_{0}$ une constante, $\vec{r}$ la position d'observation et $k^{*}$ le nombre d'onde équivalent de l'onde de compression

$$
k^{*}=\frac{\omega}{C_{\mathrm{p}}^{*}}+i \alpha^{*}
$$

avec $C_{\mathrm{p}}^{*}$ la vitesse de phase et $\alpha^{*}$ l'atténuation de l'onde en fonction de la fréquence dans le matériau équivalent.

$k^{*}$ est déduit de la connaissance du nombre d'onde dans la matrice $k_{l}$. Ce formalisme a été proposé dans le cas de $n_{0}$ diffuseurs sphériques identiques, mais peut être étendu à d'autres cas.

$$
\left(\frac{k^{*}}{k_{1}}\right)^{2}=\left[1+\frac{2 \pi n_{0} f(0)}{k_{1}^{2}}\right]^{2}-\left[\frac{2 \pi n_{0} f(\pi)}{k_{1}^{2}}\right]^{2}
$$

La fonction d'amplitude est calculée en fonction de l'angle d'observation $\theta$ à l'aide d'un opérateur spécifique appelé T-matrix [2]. $f(0)$ et $f(\pi)$ sont les amplitudes diffusées en avant et en arrière par un obstacle. Il est possible de moyenner sur des diffuseurs de taille ou/et de nature différentes.

Pour simuler le béton, nous introduisons des billes de verre de dimension et géométrie uniques supposées équivalentes à la valeur moyenne des dimensions et des géométries de l'ensemble des granulats.

Pour analyser l'endommagement du béton, il est nécessaire de modéliser les fissures. Des travaux récents permettent de modéliser l'onde diffusée sur une fissure [3]. En raison du nombre, de la taille et de la distribution statistique des fissures d'un béton endommagé, il est plus simple de prendre en compte la fissuration sous forme de sphère équivalente. Nous faisons donc l'hypothèse qu'un ensemble de fissures orientées aléatoirement dans l'espace aura des interactions globales équivalentes à celles d'un ensemble de billes identiques de diamètre judicieusement choisi.

Nous comparons ainsi les effets de billes de dimensions proches, soit d'air (diamètre 2,84 mm), soit de solide (3 $\mathrm{mm})$ sur les variations de vitesse et d'atténuation en fonction de la fréquence. Nous pourrons ainsi apprécier d'une façon différentielle l'importance d'un endommagement simulé au regard de granulats simulés. Une vérification expérimentale est menée en parallèle afin de valider les calculs sur un banc d'essais qui a fait l'objet de travaux antérieurs [2].

Les échantillons de $250 \mathrm{~mm}$ de diamètre, de $50 \mathrm{~mm}$ d'épaisseur sont testés en immersion afin d'assurer une saturation constante des éprouvettes. La vitesse $V$ et l'atténuation $\alpha$ des ondes ultrasonores de compression en fonction de la fréquence sont obtenues par comparaison entre le parcours dans l'eau sans éprouvette puis avec éprouvette. L'émission et la réception sont faites par trois jeux de traducteurs permettant de couvrir la gamme de fréquence jusqu'à 1,2 MHz.

Les figures 1 et 2 montrent les évolutions de la vitesse et de l'atténuation en fonction de la fréquence. Les mesures expérimentales comparées aux résultats analytiques sont proches et des amplitudes de variation tout à fait similaires. Le calcul d'atténuation pour les hautes fréquences dans le cas de billes de polystyrène diverge en valeur absolue avec les mesures. Le plateau atteint est considéré comme la limite du domaine stochastique de propagation et donc du domaine de validité du modèle d'homogénéisation. Cette limite est dépendante de la taille, la nature et la densité des diffuseurs.

Nous comparons les variations de vitesse et d'atténuation des éprouvettes de mortier (Mo) avec celles contenant des billes de polystyrène expansé, donc pleines d'air, simulant l'endommagement $\left(\mathrm{Mo}+\mathrm{Po}_{\mathrm{O}}\right)$ et celles contenant des billes de verre $(\mathrm{Mo}+\mathrm{Bi})$ simulant les granulats. Les ondes ultrasonores dans le verre étant plus rapides $(5700 \mathrm{~m} / \mathrm{s})$ que dans la matrice de mortier, la présence de billes de verre augmente la vitesse équivalente de phase des ondes. Dans le cas de billes de polystyrène expansé que nous pouvons assimiler à des billes d'air, le résultat est inverse. Les variations d'atténuation dans le cas du verre sont plus faibles que dans celui des 

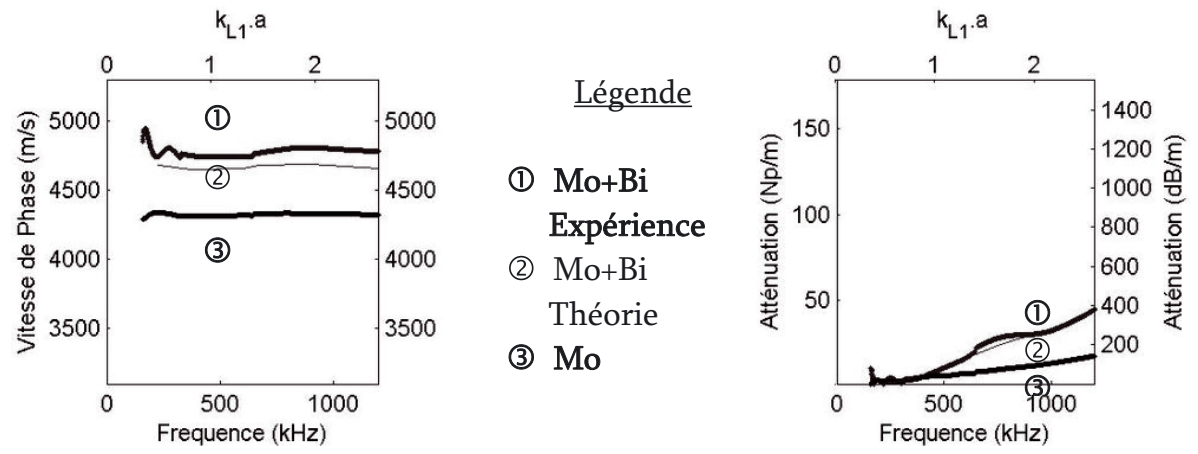

Fig. 1. $V$ et $\alpha=f$ (fréquence) : Mortier (Mo) et Mortier $+30 \%$ de billes de verre $(\mathrm{Mo}+\mathrm{Bi})$.
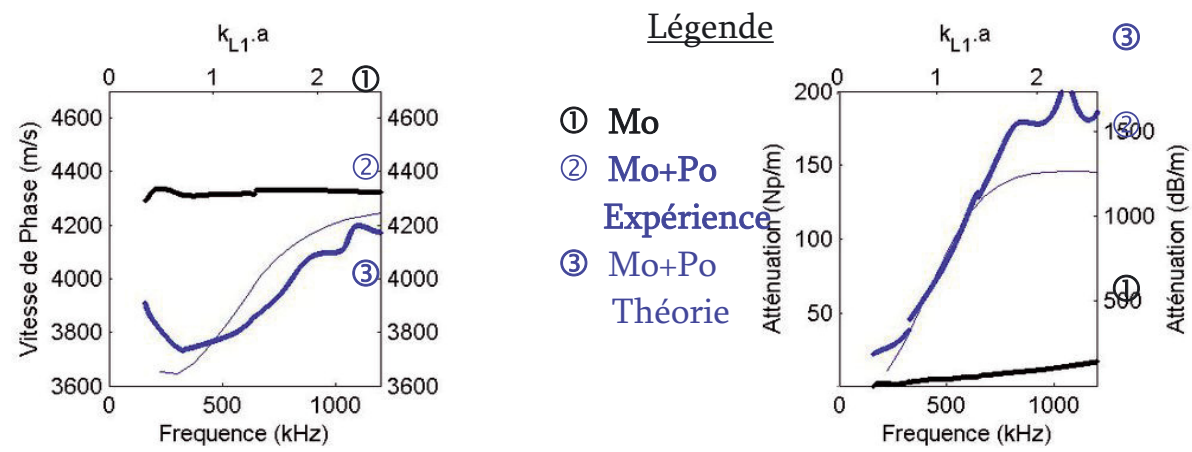

Fig. 2. $V$ et $\alpha=f$ (fréq) : Mortier (Mo) et Mortier $+30 \%$ de billes de polystyrène (Mo+Po).

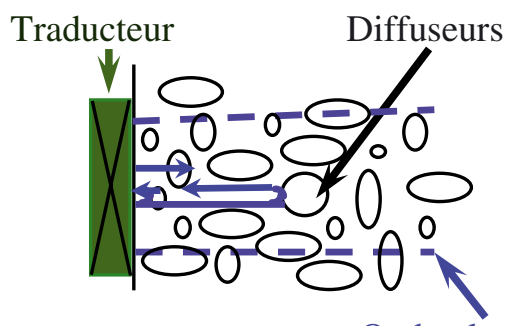

Onde ultrasonore

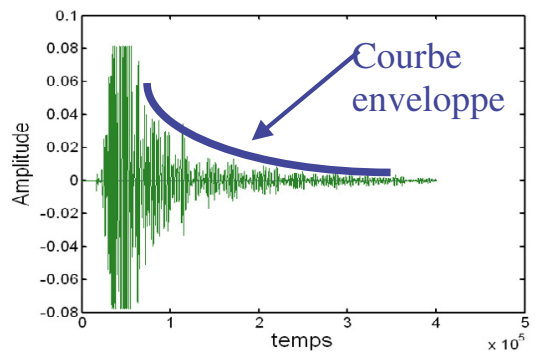

Fig. 3. Principe des ondes rétrodiffusées.

billes d'air ce qui est en accord avec les différences de contrastes acoustiques respectifs des diffuseurs.

En terme d'observables, nous constatons une sensibilité de l'atténuation des ondes ultrasonores à la présence des billes beaucoup plus importante que celle de la vitesse dans ses évolutions avec la fréquence. Ces résultats sont confirmés sur des billes de verres de $10 \mathrm{~mm}$ [4], sur des dalles en béton [5] et des bétons endommagés en laboratoire [2]. De plus en raison de la difficulté de mise en ouvre sur site en terme de couplage et de maîtrise de tous les paramètres influençant la mesure, l'atténuation est un observable plus dynamique que la vitesse à ce jour. Enfin, la fréquence la plus dynamique pour travailler est $1 \mathrm{MHz}$. Elle est liée aux résultats des figures 2 et 3 . En effet, l'atténuation est pour cette fréquence la plus sensible en termes de variations entre les éprouvettes saines simulées et endommagées simulées tout en restant dans le domaine stochastique dans lequel les phénomènes de diffusion sont les plus importants.

\section{Mesure de l'atténuation}

La mesure de l'atténuation sur site comme de la vitesse nécessite la mesure de la distance. Ce point limite directement l'estimation des paramètres acoustiques à des cas simples en terme de géométrie et d'accessibilité. L'accès à deux faces n'étant pas toujours possible et le travail par contact étant rédhibitoire pour la mesure d'une atténuation quelconque, il devient essentiel de proposer de nouvelles méthodes d'investigation. Des techniques de surface développées sur la base de traducteurs sans contact [6] ou d'ensemble de mesure laser [5] permettent d'accéder à la vitesse et l'atténuation des ondes 
de surface avec des ensembles robotisés. Ces appareillages ont pour objectif de contrôler le béton de peau d'une structure.

Une autre méthode applicable plus aisément in situ consiste à analyser la décroissance des ondes rétrodiffusées par le béton.

\subsection{Ondes rétrodiffusées}

Le béton étant particulièrement hétérogène une partie importante de l'énergie est diffusée dans l'espace. Cette partie de l'onde interagit d'une manière successive sur l'ensemble des diffuseurs situés sur son parcours. Les signaux rétrodiffusés correspondent à la somme des composantes qui retournent vers le traducteur. Ces signaux, composés de «bruit de structure » souvent éliminé en contrôle non destructif, deviennent dans notre cas porteur d'informations représentatives du matériau et de son état. L'atténuation de ces ondes rétrodiffusées est liée à la taille, densité et nature des diffuseurs ainsi que des caractéristiques de la matrice.

Le principe de génération est réalisé par un traducteur unique ou par un traducteur à deux pastilles émettrice et réceptrice séparées annulaires. L'information est intrinsèque au signal et se suffit à elle-même.

L'amplitude des contributions est décroissante dans le temps et Sanïe [7] propose un modèle pour extraire l'atténuation du signal reçu par le traducteur $r(t)$ sur la base de l'équation suivante.

$$
r(t)=u_{0} \mathrm{e}^{-\alpha C t} \sum_{k=1}^{M} \sigma_{k} \mathrm{e}^{i \phi_{k}}
$$

où $u_{0}$ est l'amplitude initiale de l'onde, $\alpha$ est l'atténuation des ondes rétrodiffusées, $C$ la vitesse des ondes dans le matériau et $t$ le temps.

$M$ définit le nombre de diffuseurs et $\sigma_{k}$ correspond à la section de diffusion liée aux caractéristiques des diffuseurs. La position temporelle $\tau_{k}$ des diffuseurs est définie $\operatorname{par} \phi_{k}=\omega\left(t-\tau_{k}\right)$.

Dans l'hypothèse d'un signal en champ lointain et de diffuseurs distribués aléatoirement en terme de dimension et de position, nous pouvons approximer la décroissance du signal par son enveloppe $-\alpha C t$. La connaissance de la vitesse conduit alors à l'extraction de l'observable $\alpha$.

\subsection{Résultats}

Deux séries d'essais ont été menées.

La première série dans le cadre du projet SENSO a pour objectif d'estimer la teneur en eau ainsi que la porosité dans un béton courant. Les mesures ont été menées sur 6 gâchées de bétons à rapports eau/ciment différents. Dans chacune d'elles, 8 éprouvettes $\left(50 \times 25 \times 12 \mathrm{~cm}^{3}\right)$ ont permis de tester le béton pour un degré de saturation de 0 puis $100 \%$ dans un premier temps, puis certaines d'entre elles ont été portées à des teneurs en eau intermédiaires

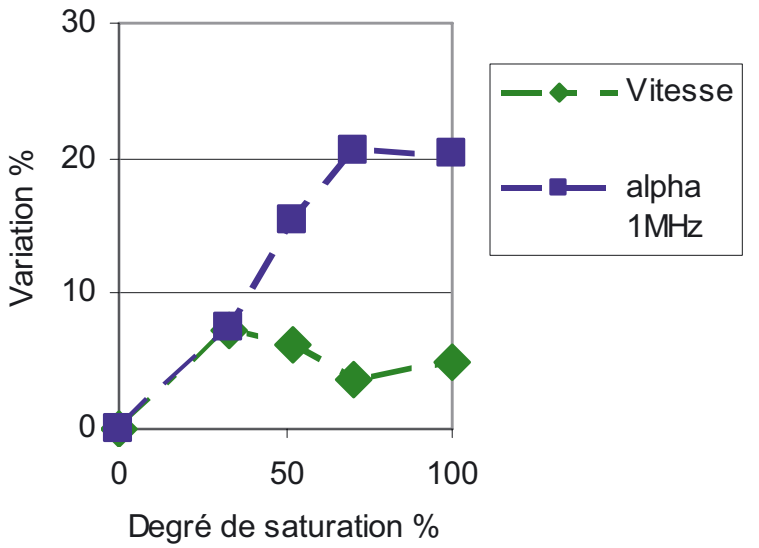

Fig. 4. Variation de l'atténuation des ondes rétrodiffusées et de la vitesse des ondes transmises pour différents degré de saturation du béton.

(33, 52 et $70 \%)$. Des mesures de l'atténuation des ondes rétrodiffusées et de la vitesse transmise ont été menées pour chaque cas d'étude.

Les variations de l'atténuation des ondes rétrodiffusées comparées à celle de la variation de la vitesse des ondes transmises sont normalisées par rapport aux valeurs obtenues pour $0 \%$. Elles montrent clairement pour des valeurs courantes de degré de saturation (plus de $50 \%$ ) que la sensibilité des ondes rétrodiffusées est de 3 à 4 fois plus importante que la vitesse transmise. Une loi de corrélation expérimentale a été déduite. Par contre, la difficulté de ces essais était de supprimer les agents de couplage liquide, pâteux ou solide. Les conditions d'essais ont donc nécessité de travailler avec une bande adhésive de protection avant de coupler. Les incertitudes de mesures que l'on exprime par le coefficient de variation CV sont alors importantes. Les valeurs de CV qui est le rapport de l'écart type de la série de mesures sur la valeur moyenne mesurée sur les essais d'une gâchée sont de 0,16 pour le coefficient d'atténuation de sondes rétrodiffusées contre 0,014 pour la vitesse transmise. Dans le cadre d'une campagne de mesures sur site avec des conditions plus faciles de couplage, nous avons réduit de $30 \%$ la valeur de ces $\mathrm{CV}$. Une étude spécifique sur les incertitudes de mesures des ondes rétrodiffusées a montré qu'il est possible de réduire par 2 voire 4 ces $\mathrm{CV}$ en optimisant les traducteurs et les méthodes d'exploitation des signaux ultrasonores.

La seconde série d'essais concerne l'endommagement thermique d'éprouvettes $(16 \times 32)$ ayant subi chacune un cycle thermique à une température spécifique $(20,80,120$, $\left.160,200{ }^{\circ} \mathrm{C}\right)$. Les mesures sont faites en immersion.

L'endommagement thermique génère des microfissures supposées isotropes qui réduisent la vitesse et augmentent l'atténuation. Les variations présentées sont normalisées par rapport aux valeurs du béton non endommagé. L'atténuation des ondes rétrodiffusées est encore une fois plus sensible que la vitesse aux variations d'endommagement. Cette sensibilité varie de 2 à 10 fois suivant la fréquence centrale du traducteur et l'état d'endommagement du béton. 


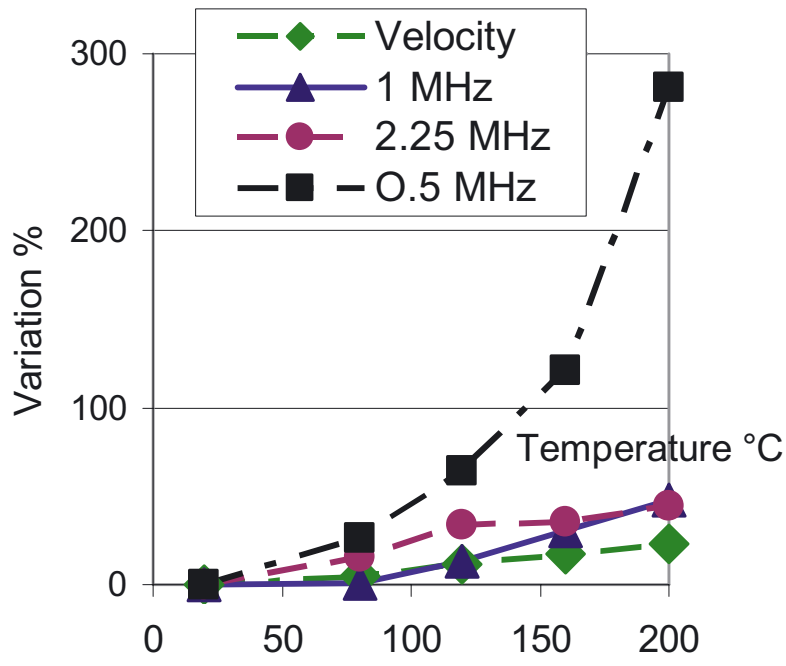

Fig. 5. Variation de l'atténuation des ondes rétrodiffusées et de la vitesse des ondes transmises pour différents niveaux d'endommagement.

\section{Conclusion}

L'évaluation non destructive du béton nécessite d'appréhender les paramètres qui influencent les procédures et mesures de contrôle. La modélisation des interactions des ondes avec les diffuseurs potentiels tels que les granulats et les microfissures nous a conduit à choisir des observables dynamiques face aux pathologies recherchées. Des essais sur des éprouvettes ont permis de confirmer que l'atténuation des ondes rétrodiffusées est plus sensible que la vitesse à la variation de teneur en eau et à l'endommagement. Cette technique est simple de mise en œuvre et conduit à des développements tant sur les aspects matériel que modélisation et exploitation des signaux.

Remerciements. Nous remercions l'ANR qui a soutenu le projet Senso.

\section{Références}

[1] P.C. Waterman, R. Truell., Multiple scattering of waves, J. Mathe. Phys. 2 (1961) 512-537

[2] J.F. Chaix, V. Garnier, G. Corneloup, Ultrasonic wave propagation in heterogeneous solid media : Theoretical analysis and experimental validation, Ultrasonic 44 (2006) 200-210

[3] M. Caleap Modélisation de la propagation d'ondes élastiques antiplanes dans des milieux multifissurés, thèse de l'Université de Bordeaux, 2009

[4] M. Rossat, Propagation des ondes ultrasonores dans les milieux hétérogènes, Application au Contrôle Non Destructif de structures en béton, Thèse de l'Université de la Méditerranée, 2007

[5] M. Chekroun, Caractérisation mécanique des premiers centimètres du béton avec des ondes de surfaces, Thèse de l'Ecole Centrale de Nantes, 2008

[6] B. Piwanowski, P. Safinowski, A. Kosecki, Contrôle non destructif du béton par ultrasons à l'aide d'un dispositif automatisé, In actes des journées COFREND, Toulouse, France, May, 17, 20, 2008

[7] J. Saniie, N.M. Bilgutay, Quantitative grain size evaluation using ultrasonic backscattered echoes, Journal of Acoustical Society of America 80 (1986) 1816-1824 